\title{
Use of Sacubitril/Valsartan in a Chronic Heart Failure Patient with Reduced Ejection Fraction Accompany Dilated Cardiomyopathy and Myocardial Indensification: A Case Report
}

Hengfen Dai ( $\nabla$ hengfendai2011@163.com )

Affiliated Fuzhou First Hospital of Fujian Medical University https://orcid.org/0000-0002-2037-4208

Yan Zhang

Fujian Medical University Affiliated Fuzhou First Hospital: Fuzhou First Hospital Jingwen Xiao

Fujian Medical University Affiliated Fuzhou First Hospital: Fuzhou First Hospital

\section{Case report}

Keywords: Heart failure with reduced ejection fraction, Dilated cardiomyopathy, Myocardial indensification, Sacubitril/Valsartan, Echocardiography features, Neuroendocrinology

Posted Date: November 19th, 2020

DOl: https://doi.org/10.21203/rs.3.rs-108070/v1

License: (c) (i) This work is licensed under a Creative Commons Attribution 4.0 International License. Read Full License 


\section{Abstract}

Rationale: Dilated cardiomyopathy (DCM) is a progressive cardiac disease characterized by ventricular dilation and contractile dysfunction and is the third most common cause of heart failure and the most common cause of heart transplantation. Heart failure (HF) and atrial fibrillation (AF) often coexist and share a mutually beneficial relationship. The presence of atrial fibrillation increases the tendency for heart failure, which can worsen its severity and increase the risk of stroke. DCM $\square \mathrm{AF}$ and HF are causal to each other in pathophysiological view. However, how these pathogens translates upon acute decompensation heart failure(ADHF) is unknown. Control acute attack of chronic heart failure is the first step of treatment.

Case summary: Herein, we described a 68-year-old man with acute decompensated heart failure (ADHF) with severe DCM and atrial fibrillation who was treated with Sacubitril/Valsartan and had a reduced ejection fraction. The patient's echocardiography features had a significant improvement under taking Sacubitril/Valsartan. Sacubitril/Valsartan may act as an intermediary that balancing of the good and the bad neuroendocrine response.

Discussion: DCM is a major cardiomyopathy and a major cause of heart transplantation. DCM's clinical prognosis is poor, additionally, along with myocardial indensification, early diagnosis and treatment is helpful to prolong the patients' life. The emergence of Sacubitril/Valsartan represents the advent of a new strategy for treating HFrEF. Its beneficial effects are related in part, at least, to an improvement of echocardiographic features and positive modulation of the neuroendocrine response to HF.

\section{Learning Points}

Sacubitril/Valsartan is the newest neurohormonal agent approved for therapy in heart failure with reduced ejection fraction (HFrEF). Dilated cardiomyopathy due to heart failure is frequent in patients with myocardial indensification and is associated with a reduced functional class and poor prognosis. The use of Sacubitril/Valsartan has been confirmed a short-term effect in this case.

\section{Introduction}

Dilated cardiomyopathy is characterised by left ventricular dilation that is associated with systolic dysfunction, with diastolic dysfunction and impaired right ventricular function can develop, and has being a major problem that lead to HF ultimately. Patients on long-term treatment sometimes have acute decompensated heart failure. Other life-threatening risks include ventricular arrhythmia, atrioventricular block, syncope, and sudden death. The extremely high hospitalization rate and potential heart transplant requirements result in a huge cost burden ${ }^{[1]}$. The focus of DCM treatment is to improve cardiac efficiency and reduce mechanical stress. The treatment of arrhythmia and the prevention of sudden death are still the main contents of treatment.

Sacubitril/Valsartan (formerly LCZ696) is a class of first-class angiotensin receptor blocking-neprilysin inhibitors (ARNI). It also inhibits the activation of RAAS by blocking angiotensin II type 1 receptor, and by 
inhibiting neprilysin (enzyme responsible for degradation) to enhance vasoactive peptides including NPs [2]. Based on the PARADIGM-HF randomized controlled trial, Sacubitril/Valsartan was approved by the US Food and Drug Administration and the European Medicines Agency in $2015^{[3]}$. Subsequently, Sacubitril/Valsartan is recommended for the treatment of chronic heart failure with reduced ejection fraction. Despite the best treatment in accordance with international guidelines(2016 ESC guidelines ${ }^{[4]}, 2017$ ACC/AHA/HFSA guidelines update ${ }^{[5]}, 2017$ CCS guidelines update ${ }^{[6]}$,all as I class of recommendation), there are still symptoms. Indeed, with the support of recent research results and a huge clinical development project, this type of new drugs for the management of heart failure may lead to a change in the concept of heart failure treatment, from inhibiting RAAS and SNS to a more comprehensive

target. To balance the neurohormonal disorders of heart failure ${ }^{[7]}$. We reported a case of an II-III New York Heart Association (NYHA) Class, stage D HFrEF in DCM's patient with myocardial indensification occurred despite optimal medical therapy and successfully treated with sacubitril/valsartan once reached persistent hemodynamic stabilization. In this case, we focus on the patient 's treatment process, clinical index, therapeutic effect and prognosis in the course of using Sacubitril/Valsartan.

\section{Case Presentation}

A 68-year-old Chinese-Han man was hospitalized for acute onset of chronic heart failure as a consequence of DCM. He had a history of coronary atherosclerotic heart disease, DCM, thyrotoxicosis, arrhythmology (frequent ventricular premature beats $\square$ persistent atrial fibrillation), cerebral infarction, type 2 diabetes,chronic HFrEF (NYHA III class). His first echocardiography, 3 years prior to presentation, found a left ventricular end-diastolic diameter (LVEDD) at $76.6 \mathrm{~mm}$, left ventricular ejection function (LVEF) at $27.6 \%$, restrictive left systolic and diastolic dysfunction, pulmonary blood pressure (BP) at $25.3 \mathrm{mmHg}$, pericardial effusion (small amount) and cardiomegaly. The patient refused to be transferred to another hospital for cardiac surgery (severity, heart transplantation), but opted for medication.

Sacubitril/Valsartan would have been available at major drugstores in the spring of 2018, as soon as it was approved in July 2017.

This patient has been using this treatment protocol of "Golden Triangle" since he was diagnosed with DCM and HFrEF in early 2016. His main prescription contained metoprolol, spironolactone and furosemide without angiotensin-converting enzyme inhibition (ACEI) and angiotensin receptor blocker (ARB), because of initial blood pressure is $115 / 74 \mathrm{mmHg}$. Multiple acute coronary syndrome events requiring unplanned visit to cardiologist during 2016. New episode of ADHFrEF occurred on september 2018. Notable admission laboratoristic values included N-terminal proBNP (NT-proBNP) of $2193 \mathrm{pg} / \mathrm{mL}$ and High-sensitivity troponin T of $21 \mathrm{ng} / \mathrm{L}$, whereas transthoracic echocardiogram showed an LVEF of $18.8 \%$, LVEDD of $86.8 \mathrm{~mm}$, and left ventricular end-systolic diameter (LVESD) of $79 \mathrm{~mm}$, with extremely poor left ventricular systolic diastolic function. He was treated yet with intravenous diuretics and digoxin and discharged on optimal medical therapy (nicorandil, furosemide, metoprolol, spironolactone, and digoxin). Several hospitalizations for acute episodes of heart failure were recorded in Table 1. 
Sacubitril/Valsartan was recommended for the treatment of HFrEF and he accepted the medicine on October 7,2018.

Table 1

Timeline-Hospitalization duration of Cardiac Care Unit (CCU)

\section{Timeline Events}

2018.09.27- Progression of DCM and ADHFrEF

2018.10.08

Prescribed medication: Fructose 1, 6-diphosphate[Clopidogrel[Rosuvastatin Calcium[

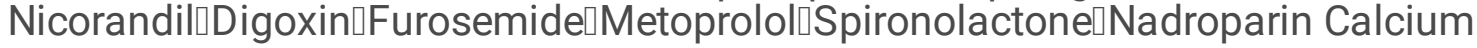

In July 2017 ,Sacubitril/Valsartan was approved for HFrEF by China food and drug administration(CFDA)

Total hospital expenses: about $1268 \$$

Introduction of Sacubitril/Valsartan

The patient accepted the new drug at a small dose $(25 \mathrm{mg}$ bid) from October-7-2018

2018.12.22- Progression of DCM,persistent AFワventricular premature beat and HFrEF

2019.01.07

Prescribed medication: Fructose 1, 6-diphosphate[Indobufen\Bumetanide[

Furosemide $\llbracket$ Metoprolol $\square$ Spironolacton $\llbracket$ Nadroparin Calcium $\square$ Methimazole $\llbracket$ Cefminox $\square$

Sacubitril/Valsartan

Total hospital expenses: about 2930 \$

2019.03.28- Acute Cerebral infarction,Progression of DCM $\triangle A F$ and HFrEF

2019.04.11

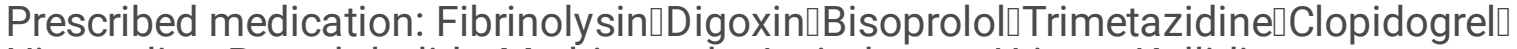

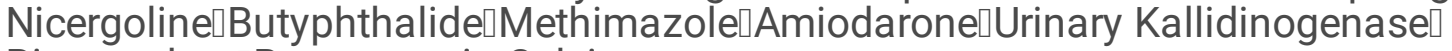

Rivaroxaban $\square$ Rosuvastatin Calcium

Total hospital expenses: about 2688 \$

2019.11.15- Progression of DCM,ADHFrEF and AF

2019.12.06

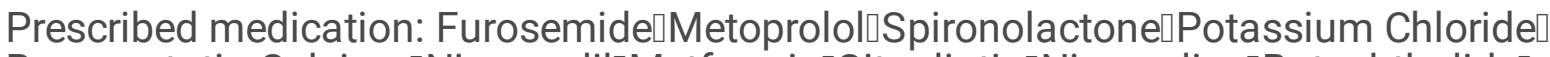
Rosuvastatin Calcium $\square$ Nicorandil[Metformin $\square$ Sitagliptin $\square$ Nicergoline $\square$ Butyphthalide $\square$

Tizanidine[Flurbiprofen Axetil[Tramadol $\square$ Cefotaxime $\square$ Moxifloxacin $\square$ Ambroxol $\square$

Terbutaline

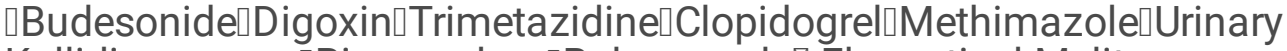

Kallidinogenase Rivaroxaban₫Rabeprazole区 Flupentixol-Melitracen

Total hospital expenses: about $11376 \$$

Physical examination was notable for blood pressure $121 / 78 \mathrm{mmHg}$, pulse $67 \mathrm{bpm}$, respiratory rate 27 apm, $\mathrm{O}_{2}$ saturation $85 \%$ on CCU indoor air with orthopneic obligatory position, and shapely figure (weight $67 \mathrm{~kg}$, height $1.71 \mathrm{~m}$ ). Arterial blood gas analysis revealed severe hypoxemia with respiratory and metabolic alkalosis ( $\mathrm{pH} 7.72, \mathrm{pO}_{2} 48 \mathrm{mmHg}, \mathrm{pCO}_{2} 24.4 \mathrm{mmHg} \mathrm{HCO}_{3}{ }^{-} 22.5 \mathrm{mmol} / \mathrm{L}$, alveolar-arterial 
gradient $32.1 \mathrm{mmHg}$ ). Although electrocardiogram highlighted atrial fibrillation with ventricular rate of $72 \mathrm{bpm}$, left atrium and left ventricular enlarged (Fig. 1), and transthoracic echocardiogram showed the same features as the previous except for LVEF of $15.86 \%$ (Fig. 2A and B). For heart failure, the patient was treated with intravenous furosemide $60 \mathrm{mg}$ q.d., Sacubitril/Valsartan $50 \mathrm{mg}$ b.i.d. (Octorber 7, 2018). On day 10, once reaching hemodynamic stabilization, Sacubitril/Valsartan midrange dose of $100 \mathrm{mg}$ b.i.d. had been added to nicorandil $5 \mathrm{mg}$ t.i.d., spironolactone $40 \mathrm{mg}$ q.d., metoprolol $47.5 \mathrm{mg}$ q.d., and digoxin $0.125 \mathrm{mg}$ q.d. But within two weeks, his BP was too low to tolerate this dose (from $121 / 78 \mathrm{mmHg}$ to $82 / 57 \mathrm{mmHg}$ ). On day 21, he kept Sacubitril/Valsartan low-range dose of $50 \mathrm{mg}$ b.i.d until November 2019, but during the period of April 2019 to May 2019, the medicine was suspended, because of high price. He administered the medicine again in consultation with the doctor of a visit in the middle of May.

On 10-months follow-up Sacubitril/Valsartan had not been increased to $100 \mathrm{mg}$ b.i.d. but remained stable at $50 \mathrm{mg}$ b.i.d. for patient's progressive clinical condition, allowing persistent and progressive clinical, laboratory (NT-proBNP reduction and High-sensitivity troponin T reduction), echocardiographic-LVEF increase $(25.55 \%$ vs $15.86 \%)$, LVEDD reduction, and LVESD reduction, and quality of life improvement without new episodes of ADHFrEF until 14-months follow-up (Fig. 2C and D; Fig. 3 echocardiographic features of all followed-up date). Because of the lack of a specific dosage regimen for Sacubitril/Valsartan in DCM-related HFrEF, we used the tolerable therapeutic dose.

HFrEF determines many neuroendocrine factors (including NT-proBNP) release through natriuretic peptide system and renin-angiotensin-aldosterone system (RAAS) ${ }^{[8]}$ (Fig. 4). Indeed, Extensive literatures have been confirming Sacubitril/Valsartan can influence pathophysiological mechanisms of patient with HF. Among sacubitril inhibits neprilysin and increase/decrease natriuretic peptides-atrial natriuretic peptide (ANP), brain natriuretic peptide (BNP), C-type natriuretic peptide (CNP), cyclic guanosine monophosphate(cGMP), aldosterone (ALD), endothelin-1(ET-1), and other factors (bradykinin, substance $P$, adrenomedullin, glucagon-like peptide, and arginine vasopressin) $[7][9,10,11,12]$, while valsartan inhibits RAAS through angiotensin II type-1 (AT1) receptor blockage. Laboratory tests indicated that these peptides' levels (mainly ANP, cGMP, ALD, ET-1) had been undulating with the course of HFrEF (Fig. 5).

\section{Discussion}

DCM is characterized by left ventricular dilation, which is related to systolic dysfunction, diastolic dysfunction and impaired right ventricular function may also occur. The treatment of DCM is mainly aimed at the treatment of heart failure symptoms, preventing disease progression and related complications, such as end-organ dysfunction, stroke, etc. Myocardial densification failure is a rare cardiomyopathy caused by arrest of normal embryogenesis of the endocardium and myocardium. The prognosis of DCM and myocardial are very different, early diagnosis and treatment is helpful to prolong the patients' life.

HFrEF due to DCM and myocardial indensification frequently complicates HFrEF. Specific therapies for DCM-related cardiomyopathy have not offered an advantage in patients with DCM-HFrEF. Here, we report 
how the use of Sacubitril/Valsartan in a patient with DCM-related HFrEF-NYHA II to III class, has resulted in a significantly improvement in clinical status and prognosis (laboratoristic, echocardiographic, and quality of life; and 14-months survival free from ADHFrEF). Before adding Sacubitril/Valsartan, the patient presented recurrent ADHFrEF despite optimal medical therapy, and more frequent of outpatient visits than after taking this medicine.

Sacubitril/Valsartan has been shown to improve mortality and reduce hospitalizations in patients with HFrEF ${ }^{[13]}$. The effect of Sacubitril/Valsartan on LVEF and reverse remodeling parameters have not been previously described by Strongly evidences. Sacubitril/Valsartan's effects were confirmed by improvement of echocardiographic features (LVEF increase, LVESD decrease, LVEDD decrease, left ventricular mass index decrease), survival free from HF (about 1 to 2 years), and quality of life ${ }^{[14,15]}$. Furthermore, compared with enalapril (ACEI), Sacubitril/valsartan had a higher effect on ADHF (HF), lower cardiovascular complications mortality and hospital stay, similar safety, and adverse reactions (such as deterioration in renal function), hyperkalemia, symptomatic hypotension, and angioedema) ${ }^{[16]}$. The echocardiographic features of this patient indicate Sacubitril/Valsartan can reverse cardiac remodeling by reducing LVEDD, LVESD, LA, RVEDD, at the same time increasing LVEF and FS.

Serological testing has been widely applied to preliminary diagnose and prognostic evaluate HF. In patients with HF, activation of the neuro-hormonal axis and inflammatory markers would take place as the disease progresses. The activation of NT-proBNP, ANP, cGMP, ALD, and ET-1 can be measured and could aid in prognostic assessments. The most specific, sensibility and widely used biomarker is NTproBNP. A rise in NT-proBNP is associated with reduced left ventricular systolic function, hypertrophy, raised filling pressures, myocardial ischaemia ${ }^{[17]}$, and has become prediction of high risk of death, need for inhospitalisation, or need for cardiac transplantation. Temporal evolution of NT-proBNP activity from administration-before to administration-after had been recorded at every follow-up time. This curve of NTproBNP-Time reflect that Sacubitril/Valsartan could be a vital medicine in reducing NT-proBNP to a certain extent. ANP and BNP act via natriuretic peptide $G$ protein-coupled transmembrane receptors activating CGMP as second messenger which, in turn, exerts natriuretic, diuretic and vasodilating action [18]. Some of the peptides substrate for neprylisin are known to play a role in HF, such as natriuretic peptides, angiotensin, endothelin, bradykinin, substance $P$, aldosterone, and arginine vasopressin. All these peptides have effects theoretically important in HF such as limiting inflammation, reducing smooth muscle contraction, neutrophil adhesion and vascular permeability both in experimental models and in humans ${ }^{[9]}$. Overall, these neuroendocrine systems interact extensively. Sacubitril/Valsartan may act as an intermediary that balancing of the good and the bad neuroendocrine response.

To our knowledge, myocardial indensification-related DCM has a poorly clinical prognosis, and causes many complications (such as ADHFrEF, arrhythmias, and systemic thromboembolism), early diagnosis and treatment is helpful to prolong the patients' life. The emergence of Sacubitril/Valsartan represents the advent of a new strategy for treating HFrEF. Its beneficial effects are related in part, at least, to an 
improvement of echocardiographic features and positive modulation of the neuroendocrine response to HF.

In conclusion, this patient benefited from Sacubitril/Valsartan in a low dose by a short-term follow-up date (14 months). Next, more studies would be need to confirm the long-term effect and projected benefit of Sacubitril/Valsartan on heart failure caused by dilated cardiomyopathy.

\section{Declarations}

\section{Ethics approval and consent to participate}

Not applicable. Due to the treatment of a single patient with approved pharmacotherapy, no ethics approval was obtained. All authors thank the patient for informed consent to this publication and the related images.

\section{Consent for publication}

The authors thank the patient for informed consent to this publication and the related information.

\section{Availability of data and materials}

The datasets used and/or analysed during the current study are available from the corresponding author on reasonable requests.

\section{Competing interests}

The authors declare that they have no competing interests.

\section{Funding}

This work was supported by the grant from Startup Fund for Scientific Research of Fujian Medical University (2017XQ1206), Fuzhou Science and Technology Project Funds (2018-S-105-2) and Fuzhou Health and Family Planning Science and Technology Innovation Platform Construction project (2018-Swp1).

\section{Authors' contributions}

Conceptualization: Hengfen Dai.

Data curation: Yan Zhang, Jingwen Xiao, Hengfen Dai.

Formal analysis: Hengfen Dai and Yan Zhang.

Investigation: Hengfen Dai and Jingwen Xiao.

Methodology: Yan Zhang, Jingwen Xiao. 
Supervision: Hengfen Dai and Yan Zhang.

Writing - original draft: Hengfen Dai and Yan Zhang.

editing: Hengfen Dai, Yan Zhang.

\section{Acknowledgements}

The authors thank patients for their informed consent to this publication and related hospitalization information.

\section{References}

1. Jefferies J L, Towbin J A. Dilated cardiomyopathy[J]. The Lancet, 2010, 375(9716): 752-762.

2. Jhund $P S$, McMurray $J \mathrm{~J} V$. The neprilysin pathway in heart failure: a review and guide on the use of sacubitril/valsartan[J]. Heart, 2016, 102(17): 1342-1347.

3. McMurray J J V, Packer M, Desai A S, et al. Angiotensin-neprilysin inhibition versus enalapril in heart failure[J]. New England Journal of Medicine, 2014, 371(11): 993-1004.

4. Ponikowski P, Voors A A, Anker S D, et al. 2016 ESC Guidelines for the diagnosis and treatment of acute and chronic heart failure: The Task Force for the diagnosis and treatment of acute and chronic heart failure of the European Society of Cardiology (ESC). Developed with the special contribution of the Heart Failure Association (HFA) of the ESC[J]. European journal of heart failure, 2016, 18(8): 891975.

5. Yancy C W, Jessup M, Bozkurt B, et al. 2017 ACC/AHA/HFSA focused update of the 2013 ACCF/AHA guideline for the management of heart failure: a report of the American College of Cardiology/American Heart Association Task Force on Clinical Practice Guidelines and the Heart Failure Society of America[J]. Journal of the American College of Cardiology, 2017, 70(6): 776-803.

6. Ezekowitz J A, O'Meara E, McDonald M A, et al. 2017 Comprehensive update of the Canadian Cardiovascular Society guidelines for the management of heart failure[J]. Canadian Journal of Cardiology, 2017, 33(11): 1342-1433.

7. Volpe M, Carnovali M, Mastromarino V. The natriuretic peptides system in the pathophysiology of heart failure: from molecular basis to treatment[J]. Clinical Science, 2015, 130(2): 57-77.

8. Solomon S D, Rizkala A R, Gong J, et al. Angiotensin receptor neprilysin inhibition in heart failure with preserved ejection fraction: rationale and design of the PARAGON-HF trial[J]. JACC: Heart Failure, 2017, 5(7): 471-482.

9. Ferrari R, Cardoso J, Fonseca M C, et al. ARNIs: balancing "the good and the bad" of neuroendocrine response to HF[J]. Clinical Research in Cardiology, 2019: 1-12.

10. Kobalava Z, Kotovskaya Y, Averkov O, et al. Pharmacodynamic and Pharmacokinetic Profiles of Sacubitril/Valsartan (LCZ 696) in Patients with Heart Failure and Reduced Ejection Fraction[J]. Cardiovascular therapeutics, 2016, 34(4): 191-198. 
11. Díez J. Chronic heart failure as a state of reduced effectiveness of the natriuretic peptide system: implications for therapy[J]. European journal of heart failure, 2017, 19(2): 167-176.

12. Bayes-Genis A, Barallat J, Richards A M. A test in context: neprilysin: function, inhibition, and biomarker[J]. Journal of the American College of Cardiology, 2016, 68(6): 639-653.

13. Packer M, McMurray J J V, Desai A S, et al. Angiotensin receptor neprilysin inhibition compared with enalapril on the risk of clinical progression in surviving patients with heart failure[J]. Circulation, 2015, 131(1): 54-61.

14. Claggett $B$, Packer $M$, McMurray $J ~ J ~ V$, et al. Estimating the long-term treatment benefits of sacubitril-valsartan[J]. New England Journal of Medicine, 2015, 373(23): 2289-2290.

15. Almufleh A, Marbach J, Chih S, et al. Ejection fraction improvement and reverse remodeling achieved with Sacubitril/Valsartan in heart failure with reduced ejection fraction patients[J]. American journal of cardiovascular disease, 2017, 7(6): 108.

16. Velazquez E J, Morrow D A, DeVore A D, et al. Angiotensin-neprilysin inhibition in acute decompensated heart failure[J]. New England Journal of Medicine, 2019, 380(6): 539-548.

17. Tschöpe $C$, Kašner $M$, Westermann $D$, et al. Elevated NT-ProBNP levels in patients with increased left ventricular filling pressure during exercise despite preserved systolic function[J]. Journal of cardiac failure, 2005, 11(5): S28-S33.

18. Ferrari R, Malagù $M$, Biscaglia $S$, et al. Remodelling after an infarct: crosstalk between life and death[J]. Cardiology, 2016, 135(2): 68-76.

\section{Figures}




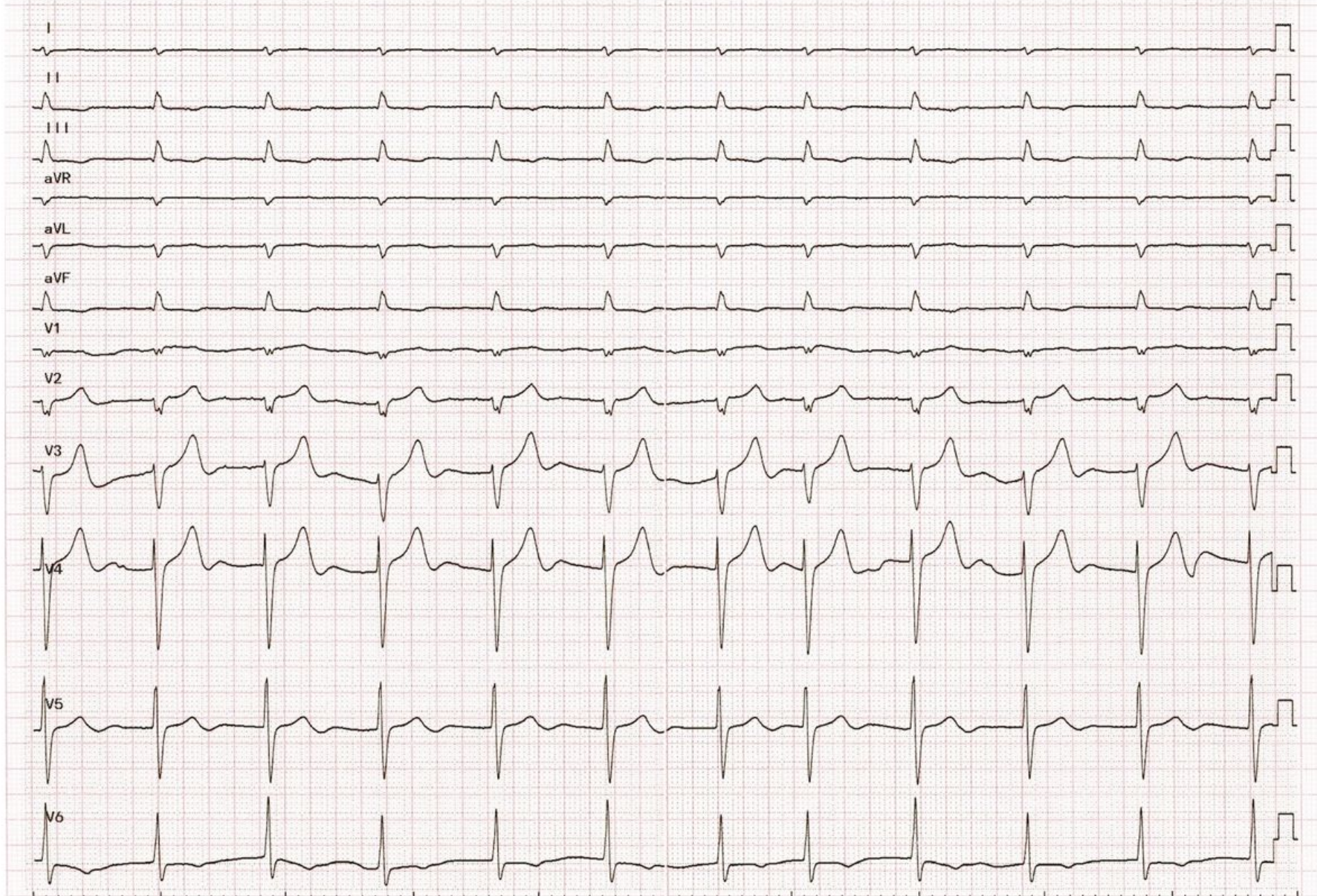

Figure 1

Electrocardiogram of the patient with atrial fibrillation, dilated cardiomyopathy and decompensated heart failure. (December 1, 2018) 


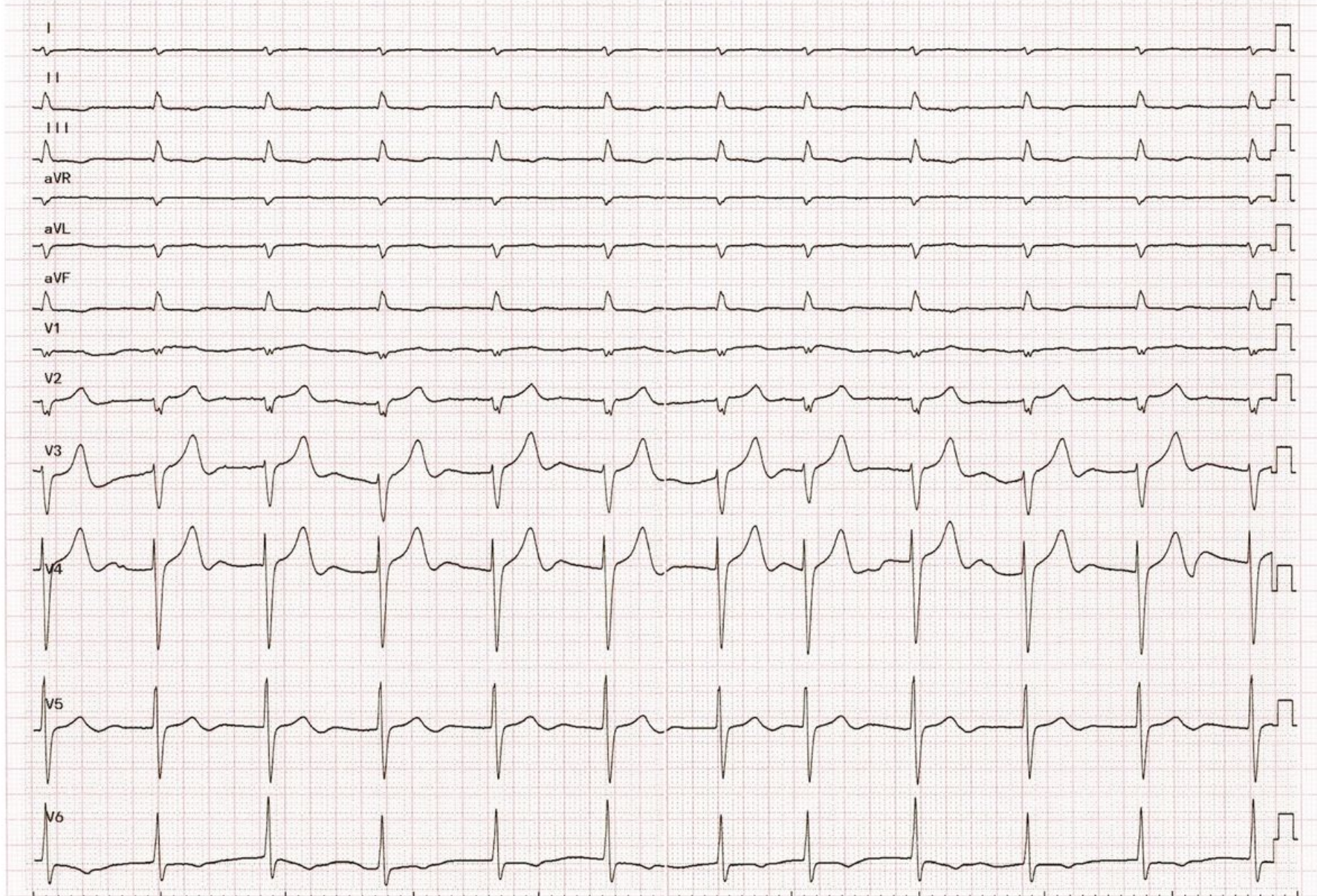

Figure 1

Electrocardiogram of the patient with atrial fibrillation, dilated cardiomyopathy and decompensated heart failure. (December 1, 2018) 


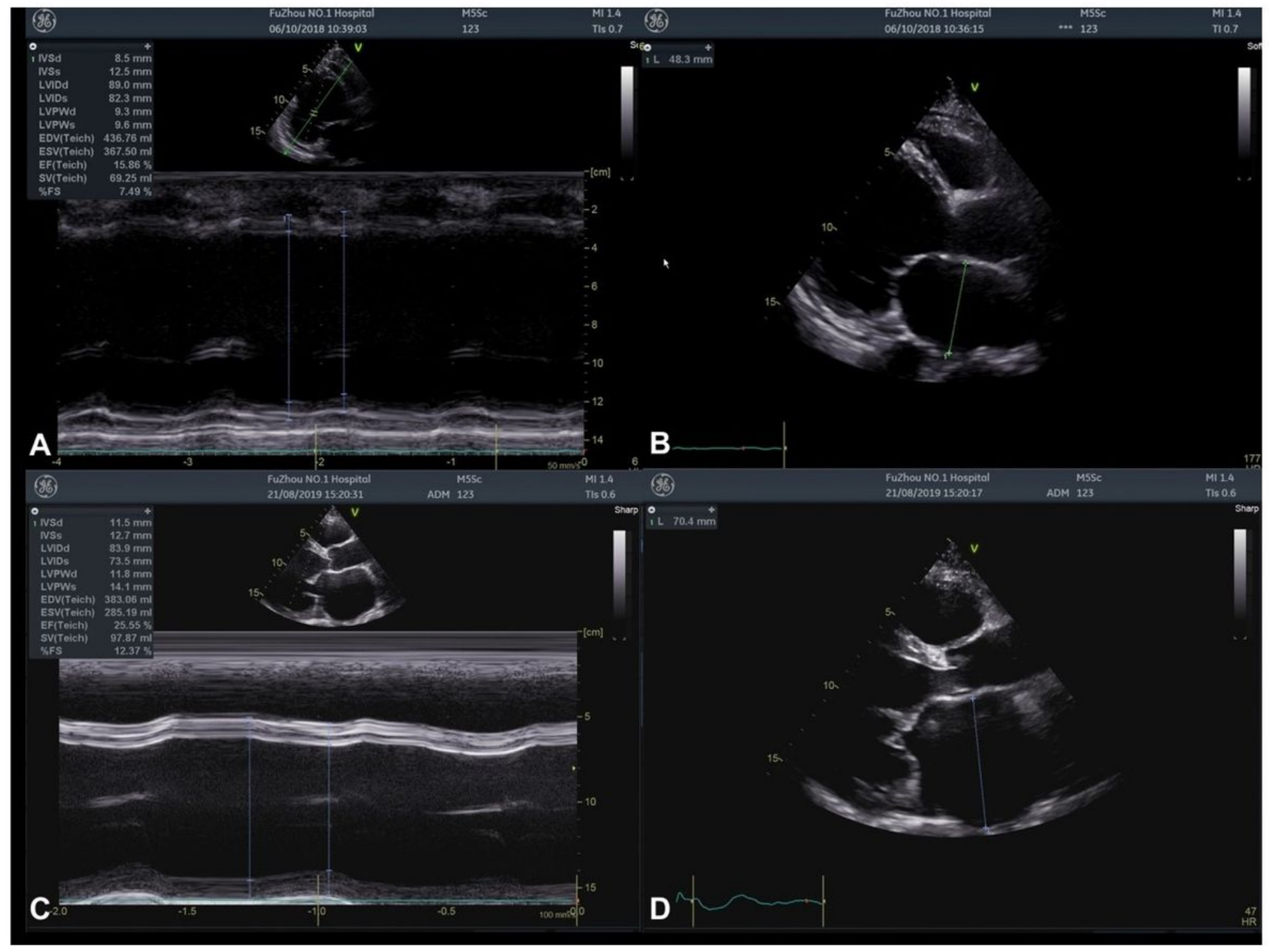

Figure 2

Echocardiographic features before (A, B) and after (C, D) 10 months of Sacubitril/Valsartan administration 


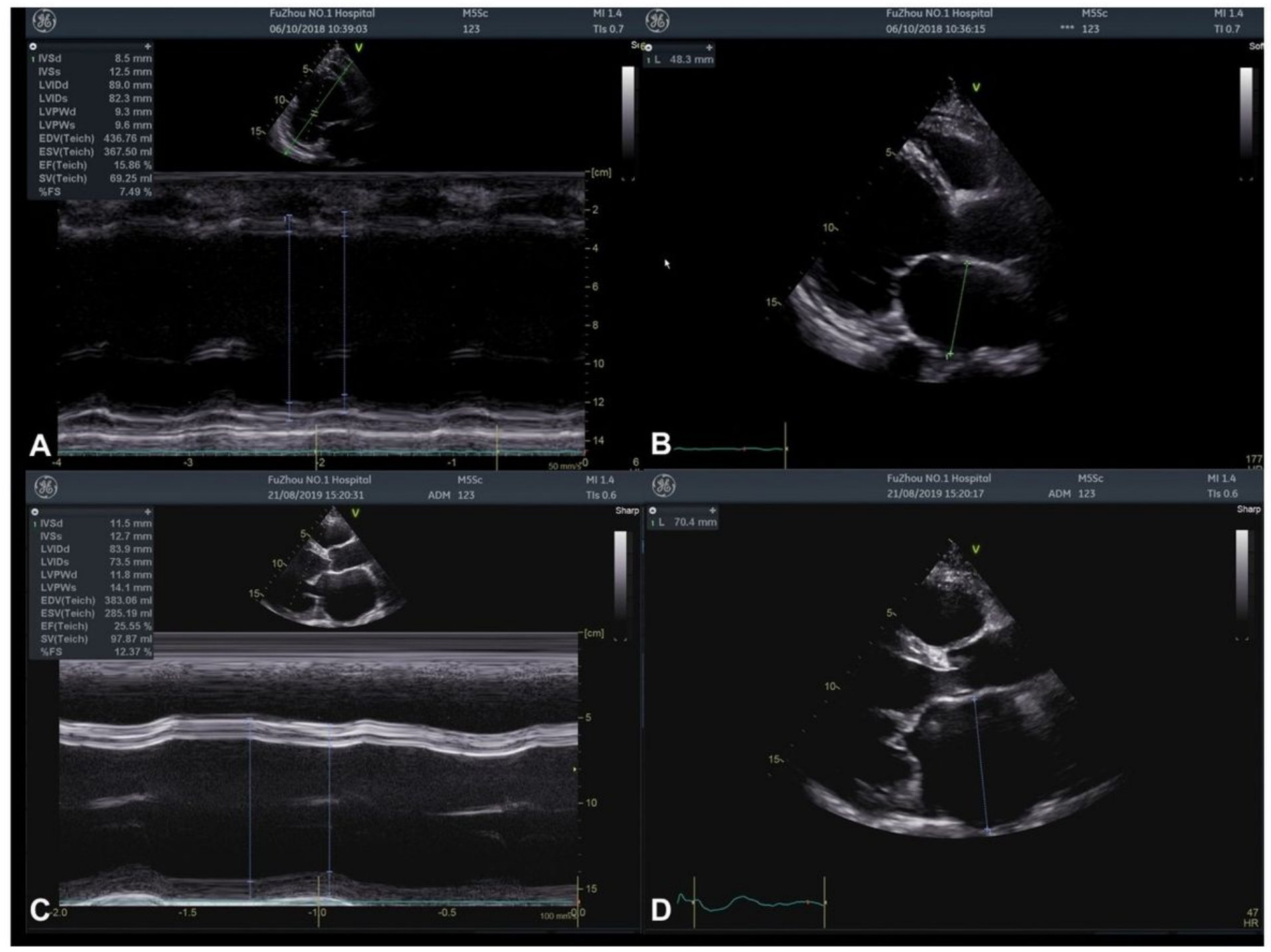

Figure 2

Echocardiographic features before (A, B) and after (C, D) 10 months of Sacubitril/Valsartan administration 


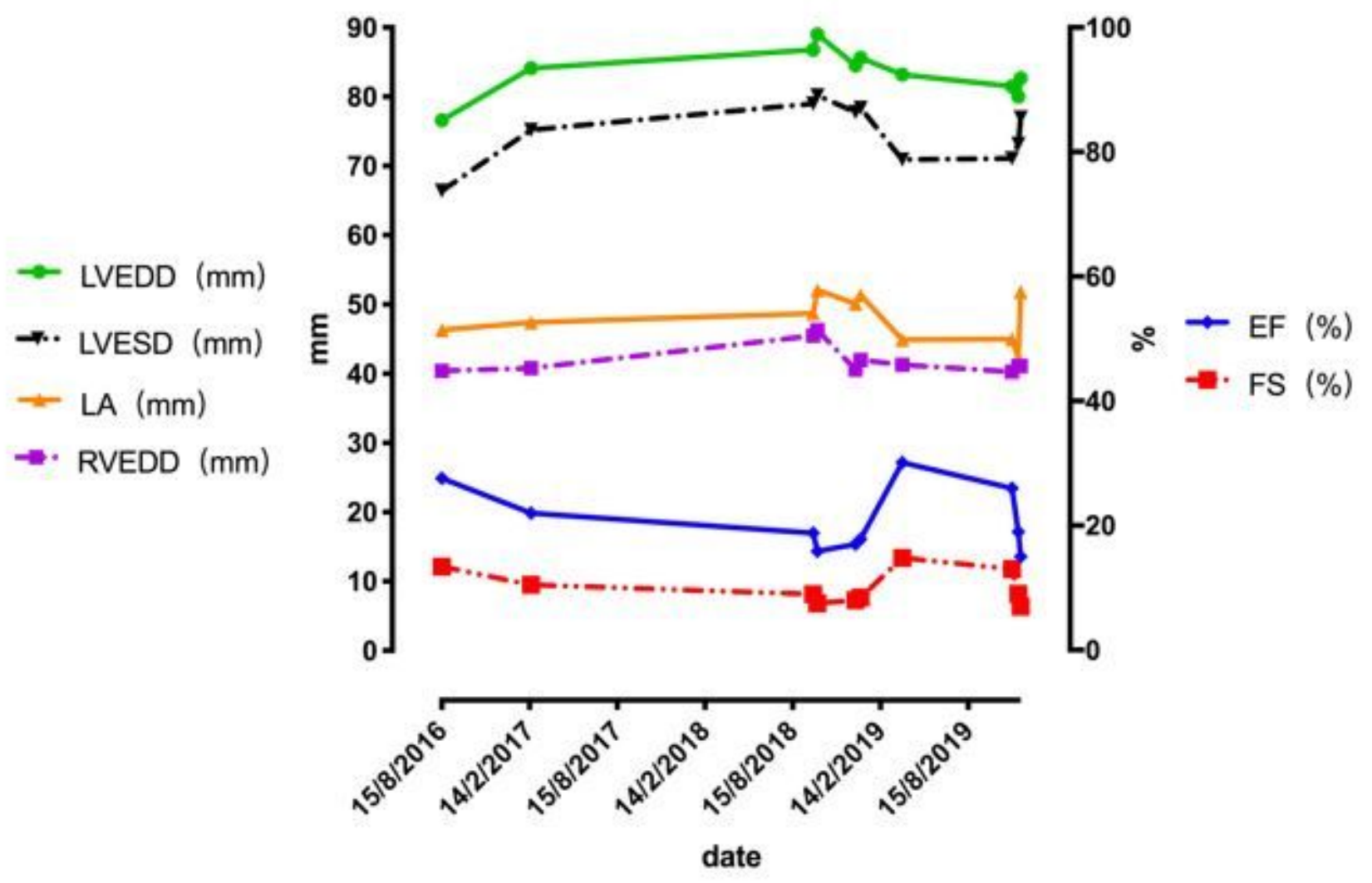

Figure 3

Echocardiographic features of different followed-up date. LVEDD = left ventricle end-diastolic diameter, LVESD = left ventricle end-systolic diameter, LA =left atrium, RVEDD = right ventricle end-diastolic diameter, LVEF = left ventricular ejection function, FS = fraction shortening. 


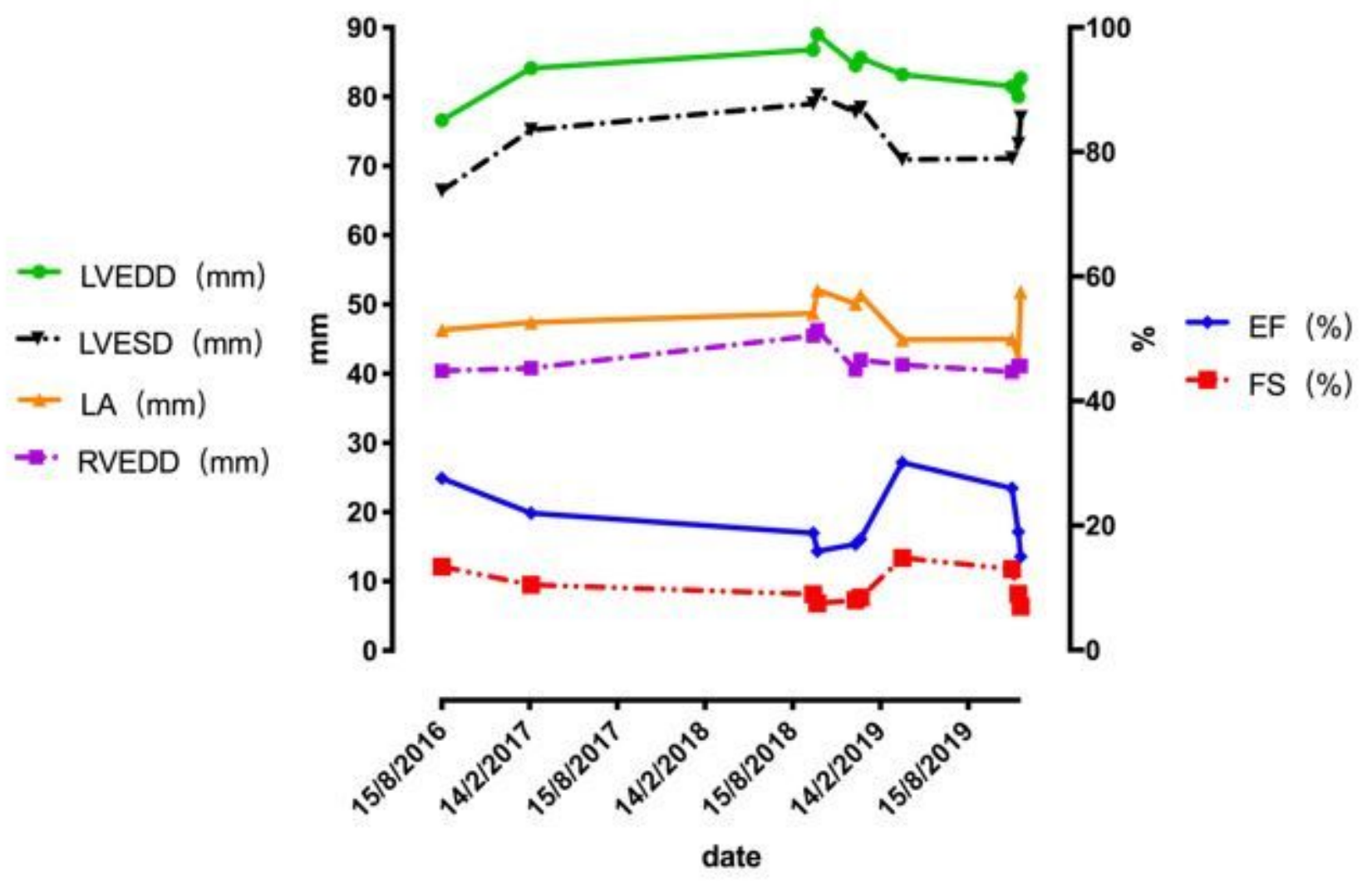

Figure 3

Echocardiographic features of different followed-up date. LVEDD = left ventricle end-diastolic diameter, LVESD = left ventricle end-systolic diameter, LA =left atrium, RVEDD = right ventricle end-diastolic diameter, LVEF = left ventricular ejection function, FS = fraction shortening. 


\section{NT-proBNP $(\mathrm{pg} / \mathrm{mL})$}

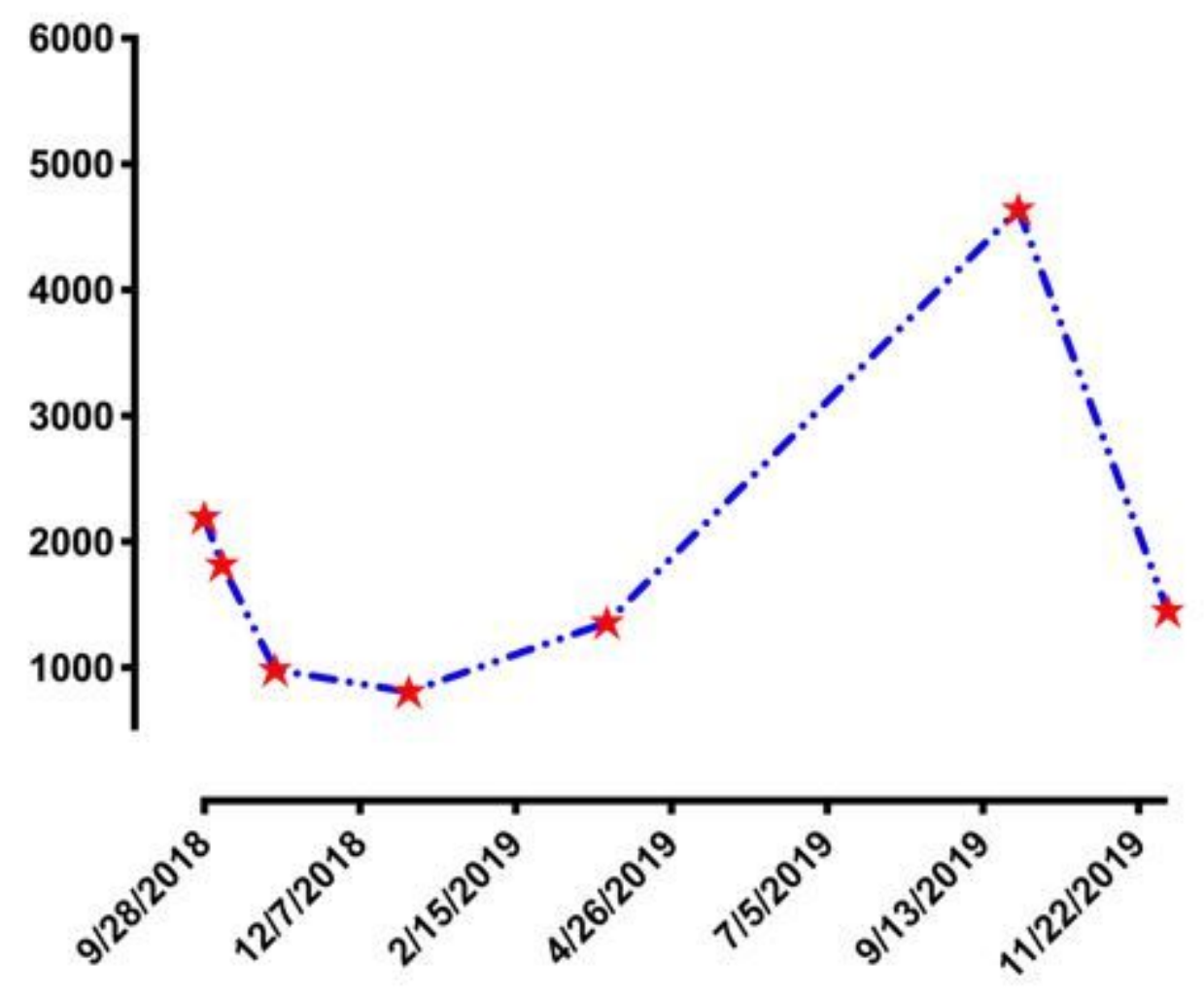

Follow-up Time

Figure 4

NT-proBNP values during the 1-week, 1-month, 3-months, 6-months, 12-months, 14months follow-up. NTproBNP $=\mathrm{N}$-terminal pro-B-type natriuretic peptide. 


\section{NT-proBNP $(\mathrm{pg} / \mathrm{mL})$}

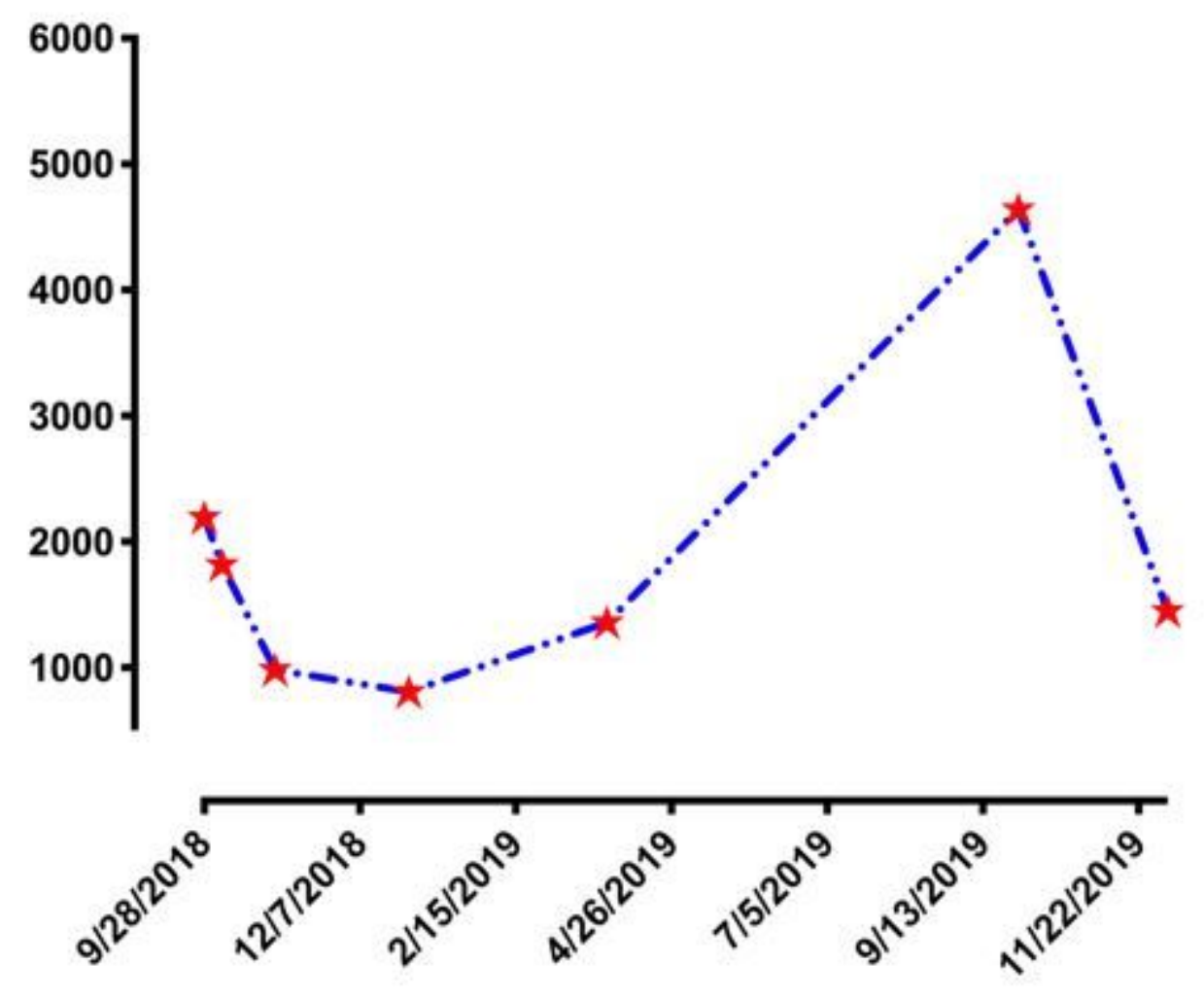

Follow-up Time

Figure 4

NT-proBNP values during the 1-week, 1-month, 3-months, 6-months, 12-months, 14months follow-up. NTproBNP $=\mathrm{N}$-terminal pro-B-type natriuretic peptide. 
Neuroendocrine factor

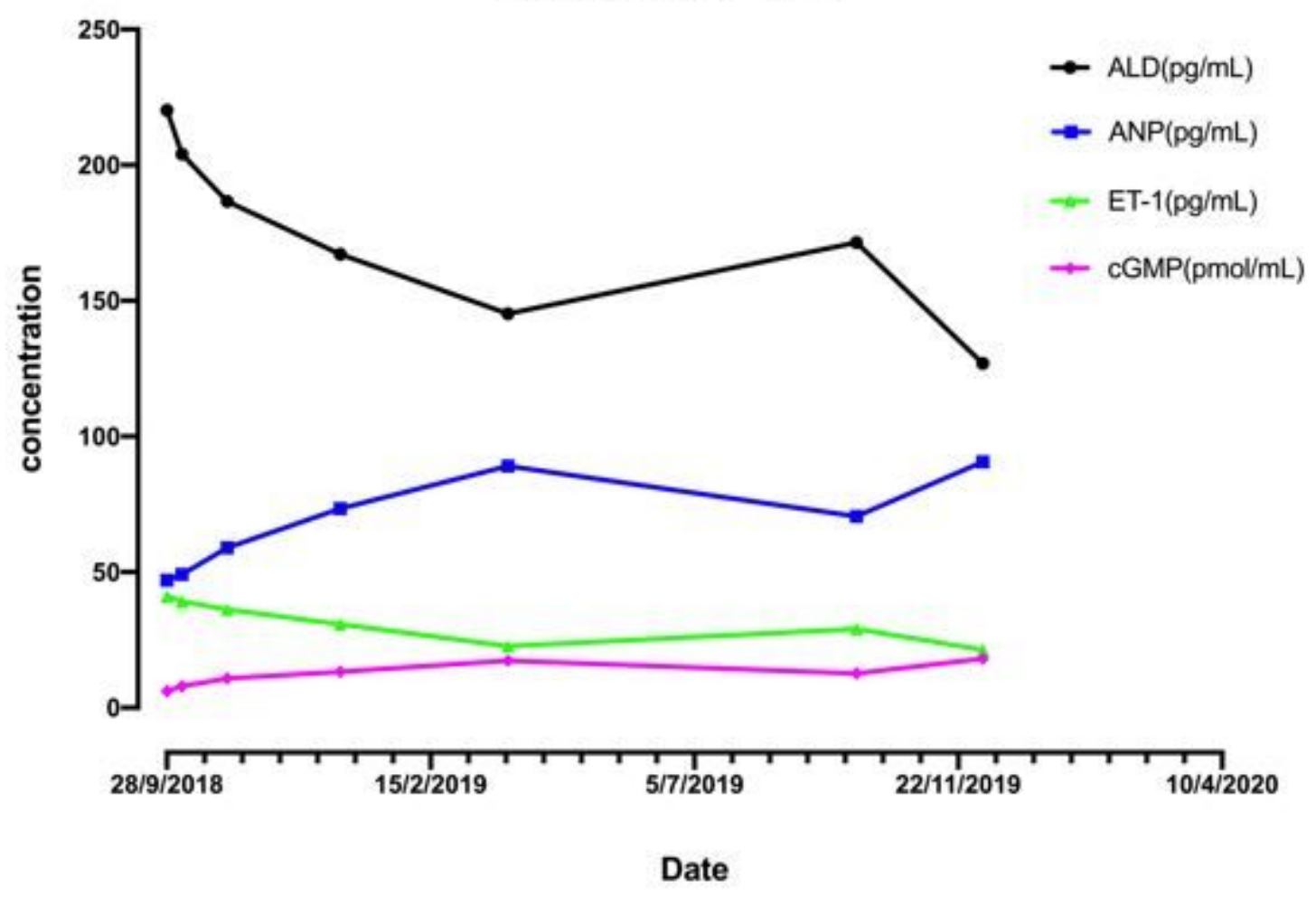

\section{Figure 5}

Concentration of neuroendocrine factors fluctuated with follow-up date. ALD = Aldosterone, ANP = atrial natriuretic peptide, ET-1 = endothelin-1, cGMP = cyclic guanosine monophosphate. 


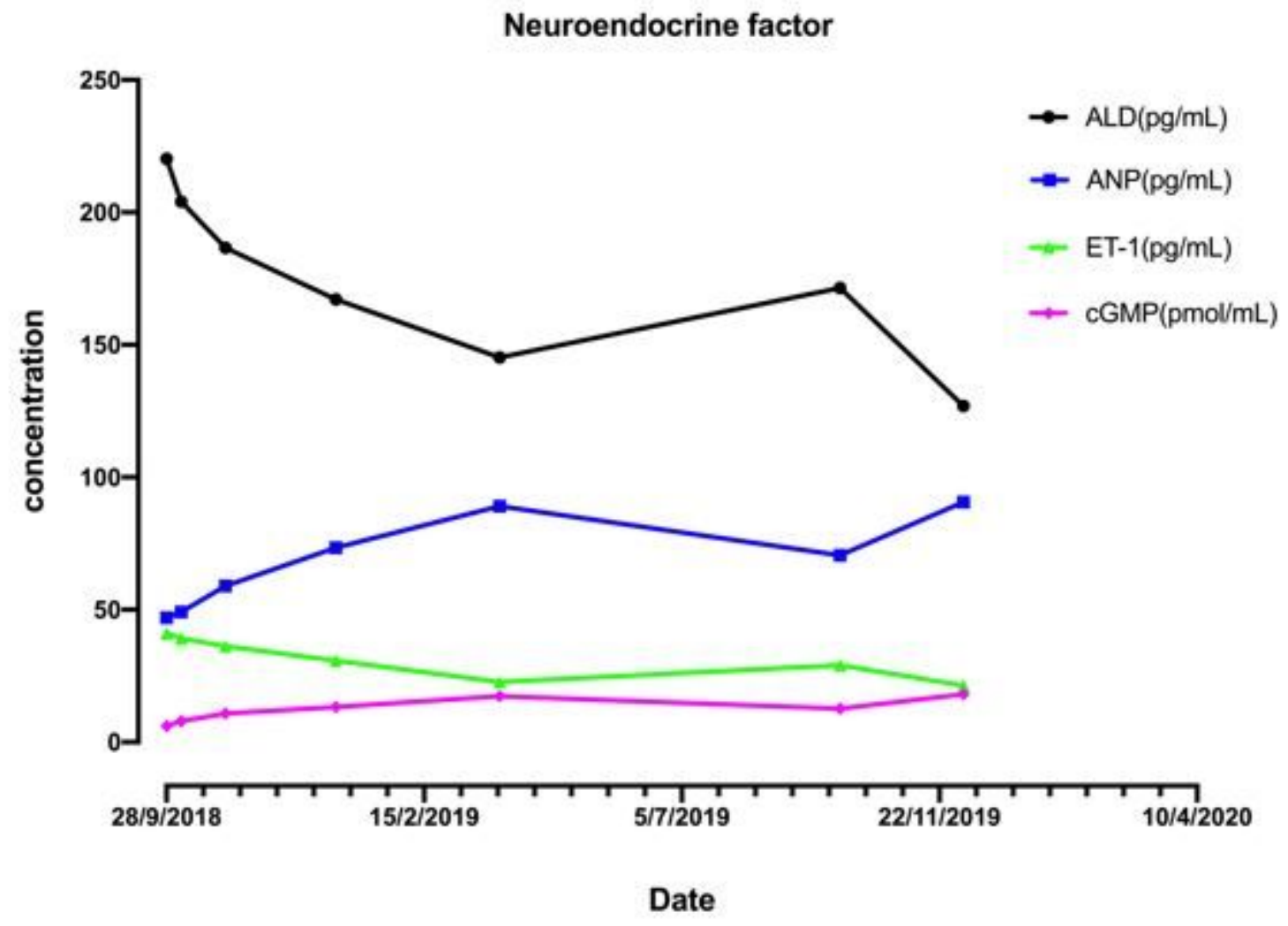

Figure 5

Concentration of neuroendocrine factors fluctuated with follow-up date. ALD = Aldosterone, ANP = atrial natriuretic peptide, ET-1 = endothelin-1, cGMP = cyclic guanosine monophosphate. 